\title{
Simple stochastic lattice gas automaton model for formation of river networks
}

\author{
Guangwu Yan, ${ }^{1, *}$ Jianying Zhang, ${ }^{1}$ Huimin Wang, ${ }^{1,3}$ and Li Guo ${ }^{2}$ \\ ${ }^{1}$ College of Mathematics, Jilin University, Changchun, 130012, China \\ ${ }^{2}$ The State key Laboratory of Nonlinear Mechanics, Chinese Academy of Science, Beijing, 100080, China \\ ${ }^{3}$ Department of Applied Mathematics, Changchun Taxation College, Changchun, 130117, China \\ (Received 4 February 2008; revised manuscript received 11 September 2008; published 4 December 2008)
}

\begin{abstract}
A stochastic lattice gas automata model for formation of river networks is proposed. The model is based on two-dimensional lattice gas automata with three fundamental principles at each node. The water source is regarded as a fixed point where a drop of water drips every time step. This system can be treated as a memory network: the probability of water moving along a direction relies on the history of the channel segment along which water drops have moved. Last, we find that the width of the river channel and the number of channels with this width meet a scaling law when the system reaches a critical status.
\end{abstract}

DOI: 10.1103/PhysRevE.78.066102

PACS number(s): 89.75.Da, 05.40.- a

\section{INTRODUCTION}

Since 1986, lattice gas automata have generated wide interest in modeling many different physical processes described generally by partial differential equations. They are also a powerful tool for modeling new physical phenomena which are not yet easily described by macroscopic equations $[1,2]$. A lattice gas system consisting of "particles" moving on a lattice satisfies certain symmetry requirements. Updating of the system is realized by designing microscopic rules for the moving and scattering of particles. Three important advantages of lattice gas automata are introduced: (i) All particle interactions are local. This method provides a way to utilize concurrent architectures. (ii) Because lattice gas automata operate with only integers and Boolean algebra, they require less computer storage so that the spatial and time resolution can be much higher than with other methods. (iii) This method may provide new insight into understanding the relationship between the microscopic mechanisms and the macroscopic behaviors for some complex physical systems. The lattice gas theory has been successful in modeling fluids and other systems.

In this paper, the lattice gas automata theory will be used to model the formation of river networks. As is known to all, river networks are among nature's most common fractal patterns [3]. Other examples of fractal structures are actual trees in gardens, plants, and cardiovascular systems in the human body, etc. Fractal structures generally satisfy some power laws or scaling laws as stated in Ref. [3]. Phenomenological scaling laws provide guidelines for the research of fractals of river networks. Any proposed model for river networks ought to be tested against these laws [4,5].

The fractal properties of river networks are demonstrated usually by some models with water erosion. In such models, drainage basins of rivers evolve into fractal forms under the effect of erosional processes [6]. By modeling the process of water erosion, stationary river patterns can be obtained after evolution from randomly perturbed surfaces [7,8]. Even though the erosion rules are local, global optimization river

\footnotetext{
*Corresponding author: yangw@email.jlu.edu.cn
}

networks can be formed and the landscape will settle into the steady state [9]. Due to the important role of the erosion process, many models for the formation of river networks and evolution of the fluvial landscape include water erosion, both continuum models with a dynamical equation [10-13], and lattice models with randomness [14,15], although the erosion process plays a role only at the initial stage in the quasirandom spanning tree model [14].

Other factors like vegetation, soil cover, rainfall, etc., may have an influence on the morphologies of river networks. So models considering precipitation and avalanching are proposed $[12,16,17]$ for formation of river networks. For the evolution of the fluvial landscape, quenched randomly pinned regions play a key role in the robust emergence of aggregation patterns with a scaling behavior in agreement with that of real river basins $[15,18]$. Moreover, the morphologies on various landscapes are studied by a slope-slope correlation function in Ref. [19].

Additionally, the dynamics of river system is sometimes considered as a good example of a self-organizing complex system [20]. Especially, Scheidegger proposed a lattice model of rivers which is defined on a slope where water on a site flows randomly to either the left or right down site. The model shows critical behavior automatically like the selforganized criticality (SOC) model. When a dynamic renormalization group is used in studying the transport in driven diffusive systems, the self-organized criticality result can be obtained, which is in agreement with the scaling exponents measured in natural river networks [21].

Furthermore, optimal channel networks (OCNs) are used to investigate the topological properties of the network itself. They are fractal structures obtained by minimizing an energy functional associated with spanning trees. Such structures are the optimal configurations formed by the erosional process taking place on a landscape. By OCNs, fractal river networks are like the river in nature and even the multifractal properties of river networks can be obtained [22]. The OCN and SOC appear similar to each other; the relations between OCNs and SOC are discussed by Rinaldo et al. in Ref. [23] as the former under generic conditions and the latter on finetuning. Minimal energy dissipation models $[24,25]$ are also proposed to study the geometry of river basins. 


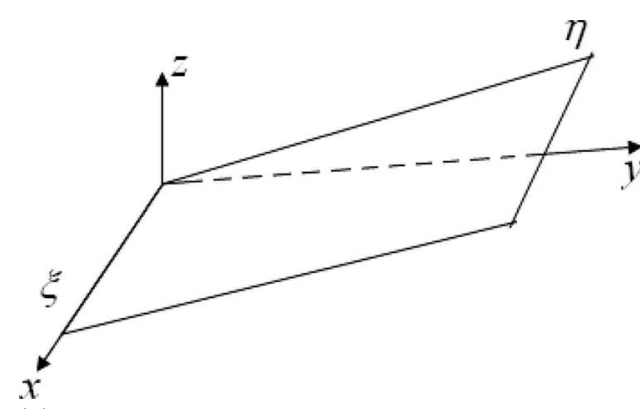

(a)

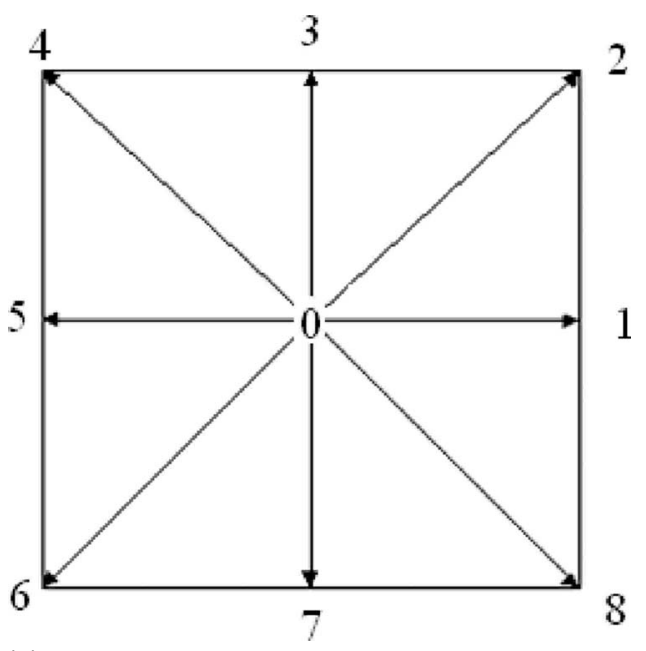

(b)

FIG. 1. A slant $\xi-\eta$ plane and (b) square 9-bit lattice.

From the studies stated above, we have a general idea of the river network formation, evolution, and its morphologies of landscape or geometry. The fractal structures of river networks are often reproduced by some erosion rules, both continuum and lattice models. In such models, a few assumptions are presented to establish a lattice model for large-scale features [10] or abstract the scaling properties through simple model [5].

In this paper, however, we present a lattice gas automata model for the formation of river networks. Instead of OCNs, our model, which is based on a few principles, is to trace the moving procedure of a drop of water along the river channels, but not consider the dissipation of energy. The model has a water source as a fixed point where a drop of water drips every time step. Namely, precipitation is only considered at the source; and vaporization is subtle while drops are in the channels. A process similar to erosion is used to obtain the fractal structure of river networks. Thus the principles we hold in the lattice model can be stated as follows: (i) Each drop of water at the node flows along the links that connect the center site to its neighbors in different probabilities. (ii) The probability of the drop of water flowing along a direction is proportional to the number of drops which have flowed along the link. (iii) The width of the river channel is proportional to the number of drops which have flowed through the segment. The above principles contain a positive feedback interaction between the probability and width of the channel. That is to say, the river channel becomes wider and wider as the drop flows. Two open problems with the lattice gas automata model are considered in this paper. The first problem concerns how to simulate the river networks formation. The second problem is that what scaling law could be obtained.

In Sec. II, a stochastic lattice gas automata model for the formation of river networks is proposed. In Sec. III, some river network patterns and the scaling laws are obtained. In Sec. IV, some conclusions are discussed.

\section{STOCHASTIC LATTICE GAS AUTOMATA FOR THE FORMATION OF RIVER NETWORKS}

\section{A. Lattice gas automata model}

Let us consider a slant $\xi$ - $\eta$ plane in three-dimensional space [see Fig. 1(a)]. A square region on this plane is selected to study the formation of river networks. A square lattice with unit spacing is used, on which each node has eight nearest neighbors connected by eight links. The water source is selected as a fixed point where a drop of water drips every time step. Here, a drop can be regarded as a particle. So a drop flowing is namely a particle moving. In the lattice, particles can only reside on the nodes or move to their nearest neighbors along these links in unit time steps. But the particle can only move along a direction down or horizontal due to gravitation. Moreover, a set of Boolean variables $S(\mathbf{x}, t)$ describing the particle occupation at node $\mathbf{x}$ and time

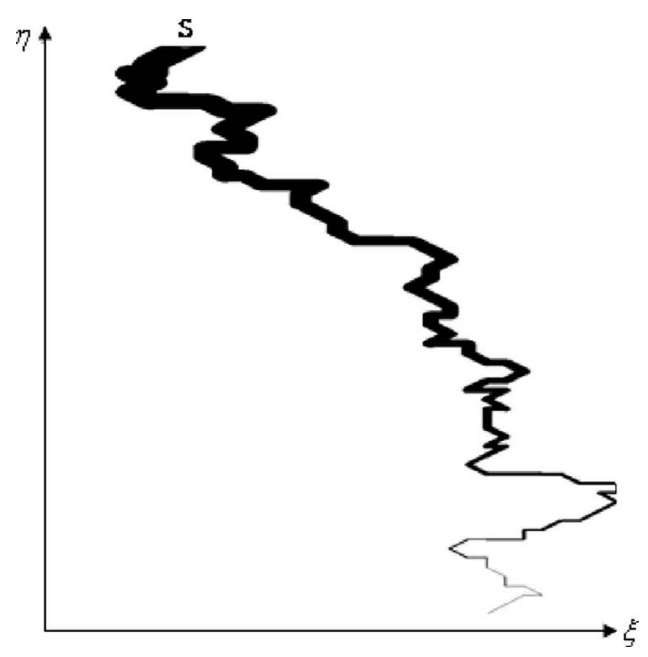

FIG. 2. The pattern of the width of the single river channel. The drop number of the water source is 100 . The lattice size is 256 $\times 256$. The water source is at point $S=(64,256) . \widetilde{P}=1, P_{0}=0.002$. 


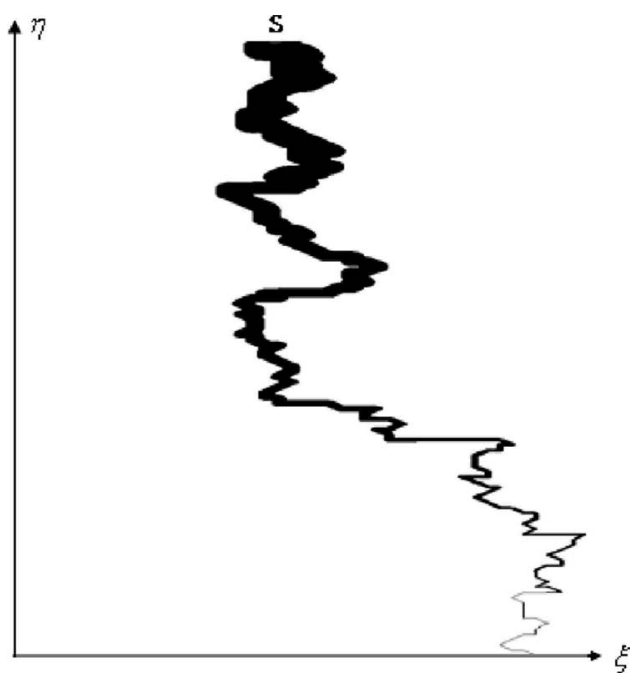

FIG. 3. The pattern of the width of the single river channel. The drop number of the water source is 200 . The lattice size is 256 $\times 256$. The water source is at point $S=(128,256)$. $\widetilde{P}=1$, $P_{0}=0.002$.

$t$ with velocity $\mathbf{e}_{\alpha}$ is defined, where $\alpha=0,1, \ldots, 8, \alpha=0$ denotes the rest particle [see Fig. 1(b)].

\section{B. Evolution rules of the lattice gas automata}

The rules are namely based on the three fundamental principles stated in Sec. I. Now, we consider the channel segment from $\mathbf{x}$ to $\mathbf{x}+\mathbf{e}_{\alpha}$. Three basic principles are stated as follows.

(i) A particle at node $\mathbf{x}$ moves along the links that connect itself to its neighbors in different probabilities.

(ii) The probability of the particle moving along the $\alpha$ direction is proportional to the number of particles which have moved along the link.

(iii) The width of the river channel is proportional to the number of particles which have moved through the segment.

According to these basic principles, a simple evolution rule of the lattice gas automata consists of two parts.

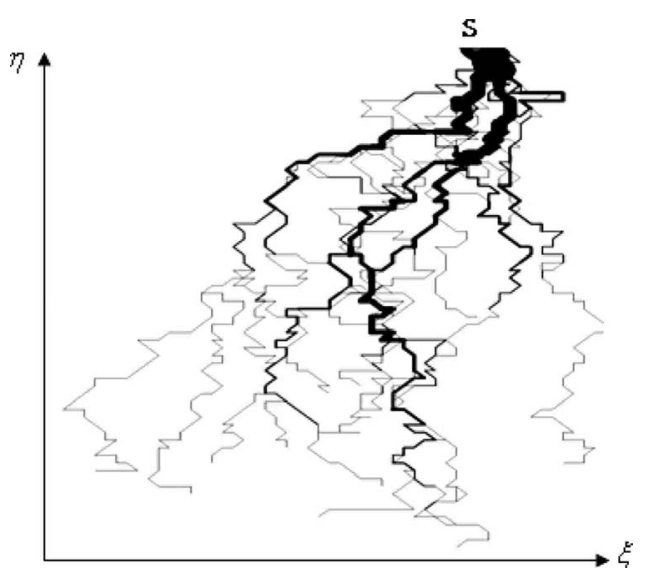

FIG. 4. The pattern of the width of the river channel with branches. The drop number of the water source is 100 . The lattice size is $256 \times 256$. The water source is at point $S=(192,256)$. $\widetilde{P}=0.95, P_{0}=0.002$.

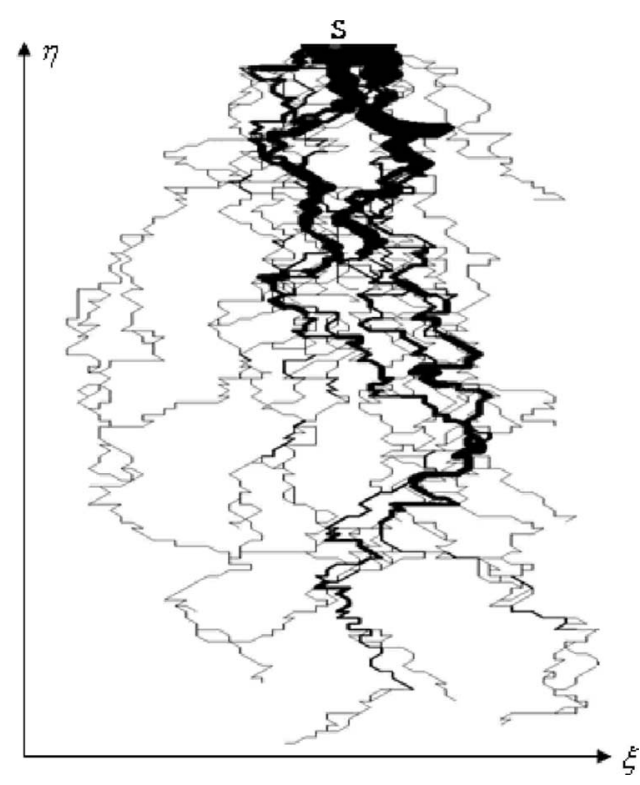

FIG. 5. The pattern of the width of the river channel with branches. The drop number of the water source is 200 . The lattice size is $256 \times 256$. The water source is at point $S=(128,256)$. $\widetilde{P}=0.95, P_{0}=0.002$.

(i) The particle moves along the $\alpha$ direction with probability $P_{\alpha}$ : namely,

$$
S\left(\mathbf{x}+\mathbf{e}_{\alpha}, t+1\right)=\left\{\begin{array}{lll}
1, & \text { if } S(\mathbf{x}, t)=1, & R \leqslant P_{\alpha}, \\
0, & \text { if } S(\mathbf{x}, t)=1, & R>P_{\alpha},
\end{array}\right.
$$

where $\alpha=1, \ldots, 8$, and $R$ is a stochastic number in the region $[0,1]$.

(ii) The particle can be vaporized in probability $P_{0}$ : namely,

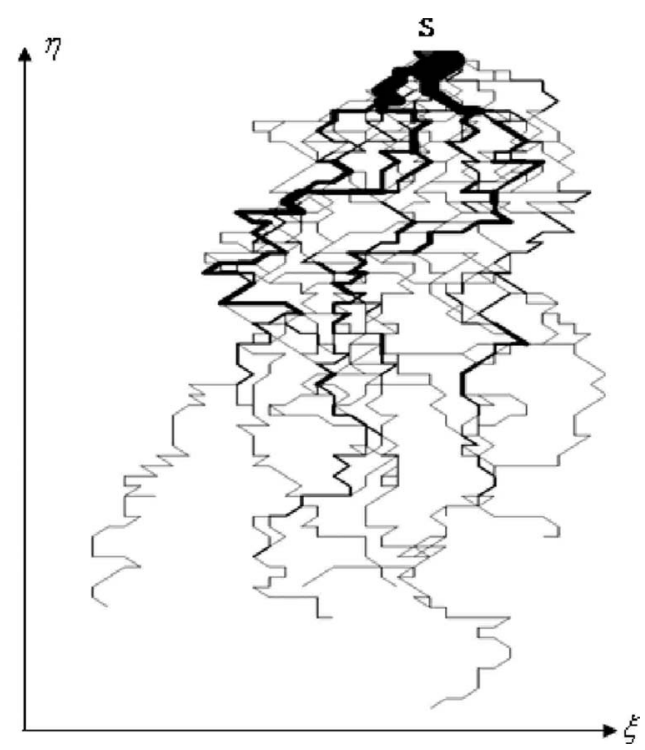

FIG. 6. The pattern of the width of the river channel with branches. The drop number of the water source is 100 . The lattice size is $256 \times 256$. The water source is at point $S=(192,256)$. $\widetilde{P}=0.90, P_{0}=0.002$. 


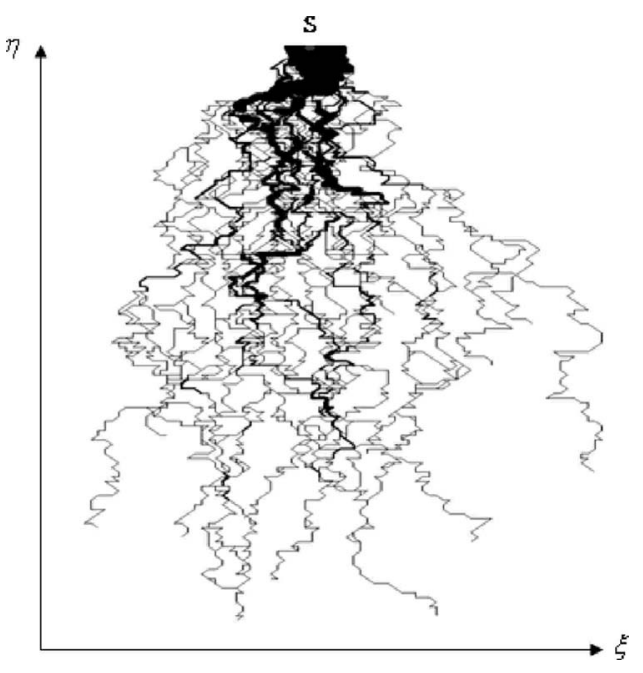

FIG. 7. The pattern of the width of the river channel with branches. The drop number of the water source is 200 . The lattice size is $256 \times 256$. The water source is at point $S=(128,256)$. $\widetilde{P}=0.90, P_{0}=0.002$.

$$
S(\mathbf{x}, t+1)=\left\{\begin{array}{lll}
0, & \text { if } S(\mathbf{x}, t)=1, & R \leqslant P_{0}, \\
1, & \text { if } S(\mathbf{x}, t)=1, & R>P_{0} .
\end{array}\right.
$$

A key problem is how to determine the probability $P_{\alpha}$. First, the particles can only move along the directions $\alpha$ $=1,5,6,7,8$ due to gravitation in the slant plane. Moreover, we assume that $P_{\alpha}$ relates to a variable $N(\mathbf{x}, T)$ defined as the sum of particles moving from $\mathbf{x}$ to its all neighbors from time $t=0$ to the present time $t=T$. It expressed as

$$
N(\mathbf{x}, T)=\sum_{\beta=1,5,6,7,8} \sum_{\tau=0}^{\tau=T} S\left(\mathbf{x}+\mathbf{e}_{\beta}, \tau\right) .
$$

The state variable $N(\mathbf{x}, T)=0$ means that there is no particle moving from $\mathbf{x}$ to its neighbors from $t=0$ to $t=T$. It shows that the region around the drop of water at $\mathbf{x}$ is dry, so the drop can flow along any direction $\mathbf{e}_{\alpha}$ while $\alpha$ $=1,5,6,7,8$ due to gravitation. Except for the vaporized drops, the probability of drops flowing to their neighbors is equal. Therefore, $P_{\alpha}=\frac{1-P_{0}}{5}$ is selected if $S(\mathbf{x}, t)=1$, and say, $1-P_{0}$ is divided equally. In general, the probability $P_{0}$ is far less than $P_{\alpha}$.

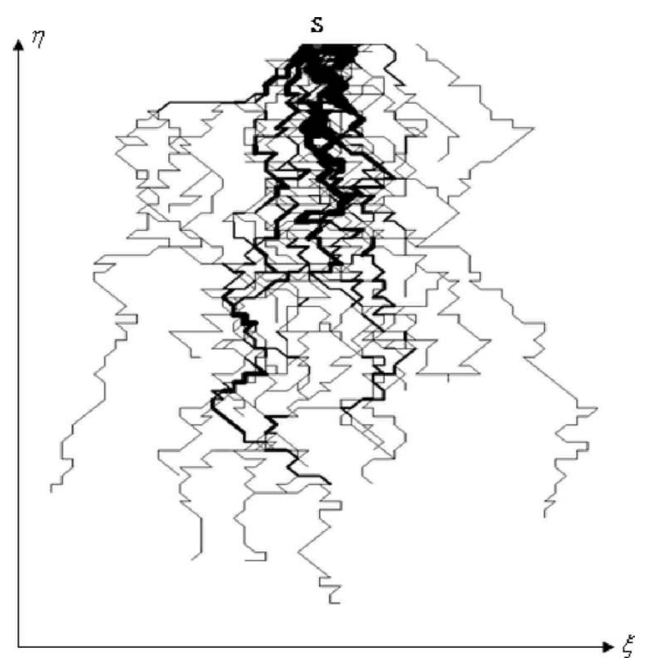

FIG. 8. The pattern of the width of the river channel with branches. The drop number of the water source is 100 . The lattice size is $256 \times 256$. The water source is at point $S=(128,256)$. $\widetilde{P}=0.80, P_{0}=0.002$.

The state variable $N(\mathbf{x}, T)>0$ means that there are some particles having moved from $\mathbf{x}$ to their neighbors from $t=0$ to $t=T$. That is to say, at least a river channel exists when a drop reaches the site $\mathbf{x}$. Therefore, the drops can flow along the channel easily.

In addition, in order to determine $P_{\alpha}$ when at least more than one channel exists, the variable $N_{\alpha}$ is defined as the sum of particles from node $\mathbf{x}$ to $\mathbf{x}+\mathbf{e}_{\alpha}$ from time $t=0$ to the present time $t=T$ :

$$
N_{\alpha}(\mathbf{x}, T)=\sum_{\tau=0}^{\tau=T} S\left(\mathbf{x}+\mathbf{e}_{\alpha}, \tau\right)
$$

Due to gravitation, $N_{\alpha}(\mathbf{x}, T)=0$ when $\alpha=2,3,4$. Thus, $N(\mathbf{x}, T)=\sum_{\alpha=1}^{\tau=8} N_{\alpha}(\mathbf{x}, T) . N_{\alpha}>0$ means that the link $\mathbf{x}$ to $\mathbf{x}$ $+\mathbf{e}_{\alpha}$ has been developed to a channel segment. According to principle (ii), the probability is directly proportional to the number of particles which have ever moved along the link; i.e., the more $N_{\alpha}$, the larger $P_{\alpha}$.

In view of what was stated above, $P_{\alpha}$ can be selected in the form

$$
P_{\alpha}= \begin{cases}\frac{1-P_{0}}{5}, & N(\mathbf{x}, t)=0, S(\mathbf{x}, t)=1, \quad \alpha=1,5,6,7,8, \\ 0, & \alpha=2,3,4, \\ \left(1-P_{0}\right)\left(\frac{1-\tilde{P}}{5}+\frac{N_{\alpha}}{N} \tilde{P}\right), & N(\mathbf{x}, t) \geqslant 1, S(\mathbf{x}, t)=1, \quad \alpha=1,5,6,7,8,\end{cases}
$$

where $\widetilde{P}$ is the probability of a particle moving along the channels which have been formed. If $\widetilde{P}<1$, the particle does not move along the formed channels. This case can cause the river to branch off. 


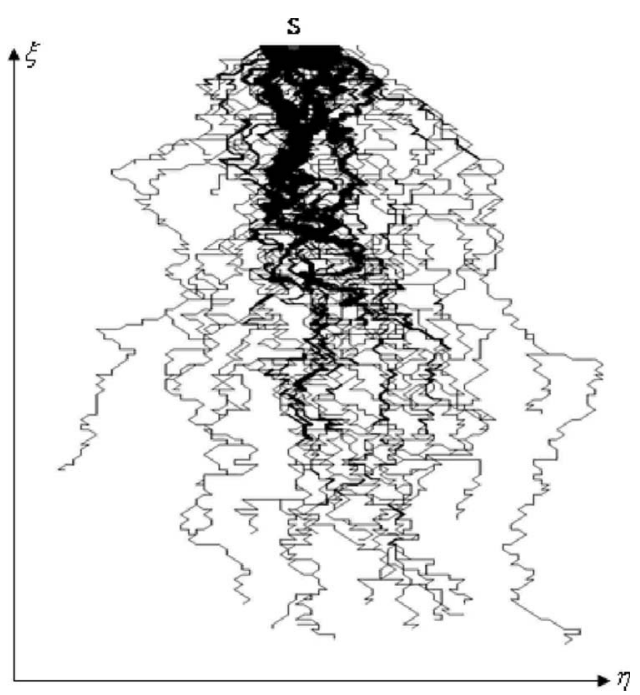

FIG. 9. The pattern of the width of the river channel with branches. The drop number of the water source is 200 . The lattice size is $256 \times 256$. The water source is at point $S=(128,256)$. $\widetilde{P}=0.80, P_{0}=0.002$.

\section{RIVER NETWORK PATTERNS AND SCALING LAWS}

\section{A. Simulation of a single river channel}

If we assume a probability $\widetilde{P}=1$, then a single river channel will form. According to principle (iii), the width of the river channel is proportional to the number of drops which have flowed through the segment. Figures 2 and 3 show the patterns of the width of the river channel in different $P_{\alpha}$ and with a different number of drops input as water source.

In the numerical simulation, the lattice size is selected as $256 \times 256$ and the probability of vaporization is selected as $P_{0}=0.002$. As to the probability $P_{\alpha}$, it is determined by Eq. (5). Namely, when $N(\mathbf{x}, t)=0, P_{\alpha}(t)=\frac{1-P_{0}}{5}, \alpha=1,5,6,7,8$, and $P_{\alpha}(t)=0, \alpha=2,3,4$. When $N(\mathbf{x}, t)>0$, it means that there exists a channel segment from site $\mathbf{x}$ to $\mathbf{x}+\mathbf{e}_{\beta}, \beta$ $\in\{1,5,6,7,8\}$, so the particle surely moves along the channel except its vaporization. The two figures show the results of the water source with 100 or 200 drops separately.

\section{B. Simulation of the river channel with branches}

If the probability $\widetilde{P}<1$, a river channel with branches can be obtained. In Figs. 4-9, we plot the patterns of the width of the river channel in different $\widetilde{P}$ and with different number of drops at water source.

In the numerical simulation, the lattice size is selected as $256 \times 256$ and the probability of vaporization is selected as $P_{0}=0.002$. The probability $P_{\alpha}$ is determined by Eq. (5). $\widetilde{P}$ can be $0.95,0.90$, or 0.80 . The water source has 100 or 200 drops.

\section{Scaling laws}

\section{Hack's law}

This river network property of drainage basin shape contains a relationship between $A$, the drainage basin area, and

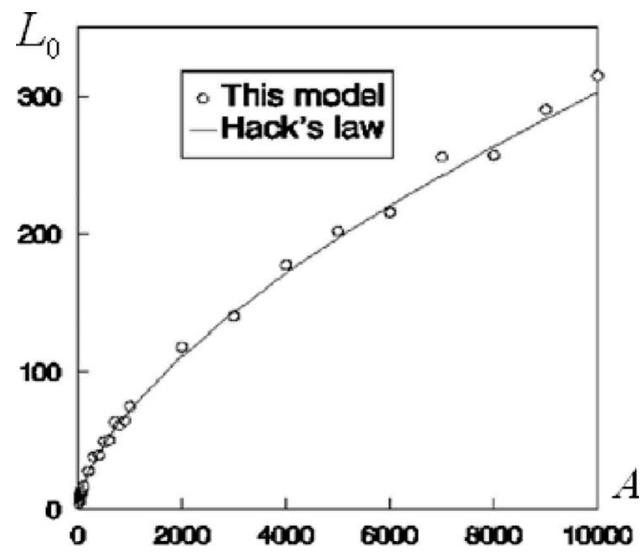

FIG. 10. The relation between the drainage basin area $A$ and main stream length $L_{0}$ in a log-log scale for this model. The line shows the relation $L_{0} \propto A^{0.62}$.

$L_{0}$, its main stream length. Hack performed a detailed investigation and found the relation to be $L_{0} \propto A^{0.6}$. We also plot the relation between the drainage basin area $A$ and the main stream length $L_{0}$ in a log-log scale for our model (see Fig. 10). The line shows the relation as $L_{0} \propto A^{0.62}$. This result is in agreement with the predicted one.

\section{Drainage basin distribution}

Let us denote $P(\geqslant A)$ as the probability that a certain randomly chosen site has a drainage basin area larger than or equal to $A$. We also plot the cumulative distributions of the drainage basin area in a log-log scale for our model in Fig. 11. The points are on a straight line in which is marked by the solid line in Fig. 11 the range from $A=10$ to $A=10^{3}$. This result shows that the cumulative distribution of the basin size follows a scaling law $P(\geqslant A) \propto A^{-0.432}$. When $A>10^{3}$, the finite-size effect becomes obvious.

\section{Horton's law}

It is known that a real river network satisfies Horton's law [27]. If we denote Horton's stream order as $\omega$, then Horton's law can be expressed as

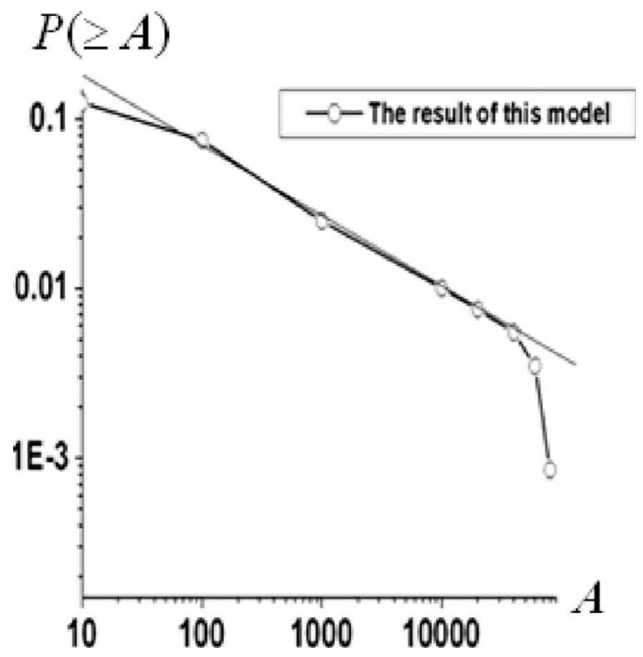

FIG. 11. Cumulative distribution of the drainage basin area $P(\geqslant A)$ in a log-log scale. The line shows a scale law as $P(\geqslant A) \propto A^{-0.432}$. 


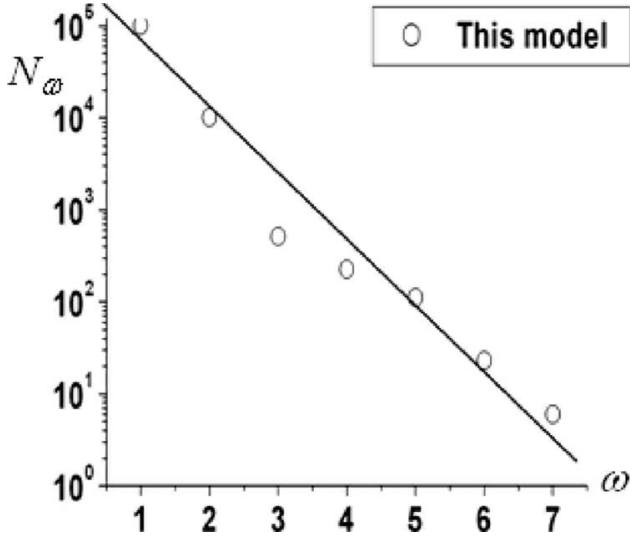

FIG. 12. Relation of the stream order $\omega$ and the number of stream branches, $N_{\omega}$.

$$
\frac{N_{\omega}}{N_{\omega+1}}=R
$$

here, $N_{\omega}$ is the number of stream branches. Using Horton's stream order, we obtain Fig. 12. From the result, we estimate the ratio as $R=4$, which is in the range of $2 \leqslant R \leqslant 6$.

\section{Relations between the width of branch $w$ and its number $m$}

Let us define $w$ and $m$ as the width of the river channel and the number of channels with width $w$, respectively. The following scaling form is expected:

$$
w=C m^{k},
$$

where $C$ is an undetermined parameter. We express Eq. (7) in logarithm form thus:

$$
\log _{10} w=D+k \log _{10} m
$$

where $D=\log _{10} C$.

Equation (8) is called the scaling law of the river channel width. It means that the width $w$ and the number of links, $m$, meet the log-log scaling law. In order to verify the form, we simulate the river channel with branches with different probabilities and water sources. Figure 13 shows the relation between the width of branch $w$ and its number $m$.

This result is similar to Hack's law. Because the lattice length is identical in unit length, the width $w$ is equal to the area with unit length of a drainage basin; the number $m$ means the length of the river basin. In fact, the scaling law, Eq. (8), is similar to Hack's law [26] in the condition of the

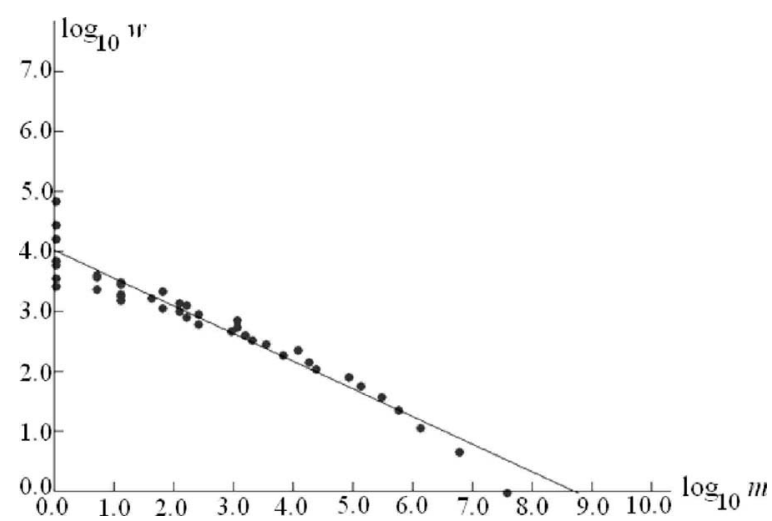

FIG. 13. The relation between the width of branch $w$ and its number $m$. The drop number of the water source is 200 . The lattice size is $256 \times 256$. The water source is at point $S=(128,256)$. $\widetilde{P}$ $=0.80, P_{0}=0.002$. The slope of the line is $k=-0.462837$.

lattice size $256 \times 256$. By some numerical simulations, we obtained that the slope of the line is in the range of $k$ $\in[-0.6,-0.4]$.

\section{CONCLUSION}

In this paper, a stochastic lattice gas automata model for formation of river networks is proposed. The model is based on two-dimensional lattice gas automata with three fundamental principles at each node. The water source is regarded as a fixed point where a drop of water drips every time step.

Two kinds of river channels are obtained by using different probability $\widetilde{P}$ : when $\widetilde{P}=1$, a single river channel appears; when $\widetilde{P}<1$, a river channel with branches appears. We also investigated Hack's law, Horton's law, and the drainage basin area distribution using our model. The results show that the scaling law is correct. On the other hand, we obtained the relation between width of branch $w$ and its number $m$.

The idea and method of this paper can be spread into a complex river network formation system. More factors may be handled if we consider a true river network system. This is what we will be working on in the future.

\section{ACKNOWLEDGMENTS}

This work is supported by the 985 Project of Jilin University, the National Nature Science Foundation of China (Grant No. 60274064), and the ChuangXin Foundation of Jilin University (Grant No. 2004CX041). We would like to thank Professor Hu Shouxin, Professor Jin Xizhuo, Dr. Yan Bing, Dr. Dong Yinfeng, Dr. Liu Yanhong, Dr. Yan Bo, and Dr. Shi Xiubo for their many helpful suggestions.
[1] U. Frisch, B. Hasslacher, and Y. Pomeau, Phys. Rev. Lett. 56, 1505 (1986).

[2] S. Wolfram, J. Stat. Phys. 45, 471 (1986).

[3] B. Mandelbrot, Fractals and Geometry of Nature (Freeman, New York 1982).
[4] A. Maritan et al., Phys. Rev. E 53, 1510 (1996).

[5] P. S. Dodds and D. H. Rothman, Phys. Rev. E 59, 4865 (1999).

[6] I. Rodriguez-Iturbe and A. Rinaldo, Fractal River Basins: Chance and self-organization (Cambridge University Press, 
Cambridge, England, 1996).

[7] H. Takayasu and H. Inaoka, Phys. Rev. Lett. 68, 966 (1992).

[8] H. Inaoka and H. Takayasu, Phys. Rev. E 47, 899 (1993).

[9] K. Sinclair and R. C. Ball, Phys. Rev. Lett. 76, 3360 (1996).

[10] S. Kramer and M. Marder, Phys. Rev. Lett. 68, 205 (1992).

[11] L. Prigozhin, Phys. Rev. E 49, 1161 (1994).

[12] A. Giacometti, A. Maritan, and J. R. Banavar, Phys. Rev. Lett. 75, 577 (1995).

[13] J. R. Banavar et al., Phys. Rev. Lett. 78, 4522 (1997).

[14] S. S. Manna and B. Subramanian, Phys. Rev. Lett. 76, 3460 (1996).

[15] G. Caldarelli, Phys. Rev. E 63, 021118 (2001).

[16] R. L. Leheny and S. R. Nagel, Phys. Rev. Lett. 71, 1470 (1993).

[17] R. L. Leheny, Phys. Rev. E 52, 5610 (1995).

[18] G. Caldarelli, A. Giacometti, and A. Maritan, Phys. Rev. E 55,
R4865 (1997).

[19] H. J. Kim and I. Kim, Phys. Rev. E 62, 3121 (2000).

[20] A. Giacometti, Phys. Rev. E 62, 6042 (2000).

[21] B. Tadić, Phys. Rev. E 58, 168 (1998).

[22] A. Rinaldo, I. Rodriguez-Iturbe, R. Rigon, et al., Phys. Rev. Lett. 70, 822 (1993).

[23] A. Rinaldo, A. Maritan, F. Colaiori, A. Flammini, R. Rignon, I. Rodriquez-Iturbe, and J. R. Banavar, Phys. Rev. Lett. 76, 3364 (1996).

[24] T. Sun, P. Meakin, and T. Jøssang, Phys. Rev. E 49, 4865 (1994).

[25] T. Sun, P. Meakin, and T. Jøssang, Phys. Rev. E 51, 5353 (1995).

[26] J. T. Hack (unpublished).

[27] R. E. Horton, Bull. Geol. Soc. Am. 56, 275 (1945). 